\title{
Diagnostic Value of the Tilt-Table Test for the Assessment of Syncope in Children and Adolescents
}

\author{
Tan Chen Wu, Derise Hadul, Maurico Scanavacca, Eduardo Sosa \\ São Paulo, SP - Brazil
}

\begin{abstract}
Objective - To assess the diagnostic value, the characteristics, and feasibility of tilt-table testing in children and adolescents.
\end{abstract}

Methods - From August 1991 to June 1997, we retrospectively assessed 94 patients under the age of 18 years who had a history of recurring syncope and presyncope of unknown origin and who were referred for tilt-table testing. These patients were divided into 2 groups: group I (children) - 36 patients with ages ranging from 3 to 12 (mean of $9.19 \pm 2.31$ ) years; group II (adolescents) - 58 patients with ages ranging from 13 to 18 (mean of $16.05 \pm 1.40$ ) years. We compared the positivity rate, the type of hemodynamic response, and the time period required for the test to become positive in the 2 groups.

Results - The positivity rates were $41.6 \%$ and $50 \%$ for groups I and II, respectively. The pattern of positive hemodynamic response that predominated in both groups was the mixed response. The mean time period required for the test to become positive was shorter in group I (11.0 \pm 7.23 min) than in group II (18.44 $7.83 \mathrm{~min})$. No patient experienced technical difficulty or complications.

Conclusion - No difference was observed in regard to feasibility, positivity rate, and pattern of positive response for the tilt-table test in children and adolescents. Pediatric patients had earlier positive responses.

Keywords: tilt-table test, neurocardiogenic syncope, children and adolescents

Instituto do Coração do Hospital de Clínicas - FMUSP

Mailing address: Denise Hachul - InCor - Av. Dr. Enéas C. Aguiar, 44 - $05403-$ 000 - São Paulo, SP, Brazil - E-mail: arritmia@incor.usp.br

English version by Stela Maris C. e Gandour
Syncope is a relatively common symptom during childhood. Prodinger et al ${ }^{1}$ reported that $15 \%$ to $50 \%$ of the adolescents have at least 1 episode of syncope by the end of adolescence. Other research carried out in university students reported a $47 \%$ prevalence of syncope in this population.

Syncope accounts for approximately 1 out of $2000 \mathrm{vi}$ sits to the pediatric emergency department ${ }^{2}$. Its incidence in children and adolescents under medical follow-up is $0.125 \%$, and it is more frequent in girls, with a peak at 15 to 19 years of age. The episode is usually isolated and relates to an acute disease or psychological stress, or both, or noxious stimuli ${ }^{3}$. In many cases, even after an extensive evaluation, the cause remains undetermined. Despite being benign most of the time, syncope usually generates anxiety and uncertainty in the patient, the family, and the environment where they live.

Syncope is a symptom with multiple causes from benign situations to etiologies with a potential risk of sudden death. Therefore, clarifying its cause is important. In children and adolescents, the neurocardiogenic etiology is supposedly the most frequent ${ }^{4}$. The tilt-table test appeared in the last decade and has been widely used for diagnosing neurocardiogenic syncope. However, controversies persist in the literature in regard to its feasibility and diagnostic value in pediatric patients ${ }^{5,6}$.

The objective of this study was to evaluate the diagnostic value, the characteristics of the results, and the feasibility of the tilt-table test in children with a history of recurring syncope of unknown origin in comparison with that in an adolescent population.

\section{Methods}

The study was carried out retrospectively. Data were collected through review of the medical records of patients under the age of 18 years who underwent tilt-table testing in the period from August 1991 to June 1997 at the laboratory of the Autonomic Evaluation of Arrhythmia Unit - Heart Institute. The study comprised patients who had had 2 or more episodes of syncope or repetitive episodes of presyn- 
cope of unknown origin, after noninvasive or invasive cardiovascular clinical and neurological evaluation, according to the requirements of each case. Ninety-four patients were selected and divided into 2 groups according to age bracket: group I, 36 patients, with ages ranging from 3 to 12 years (mean age of $9.19 \pm 2.31$ years); group II, 58 patients, with ages ranging from 13 to 18 years (mean of $16.05 \pm 1.40$ years).

The patients underwent tilt-table testing during the morning while they were fasting, in a calm, quiet, and dimly lit environment. No patient was receiving medication that might interfere with the result of the test. No sensitizing agents, venous puncture, or invasive procedures were used.

For tilt-table testing, a tilting gurney (orthostatic gurney - Carci Ind. e Com. - São Paulo, modified in the bioengineering sector of the Heart Institute) was used with maximum angulation of $60^{\circ}$ and a platform for the feet. In the horizontal dorsal decubitus position, the patients were monitored for blood pressure with a noninvasive digital monitor (2300 - Finapres - Ohmeda) and electrocardiography was performed with a polygraph Hewlett-Packard (HP). Recordings of the pressure curves, of heart rate, and of electrocardiography were performed with an HP polygraph coupled with a monitor (Sistema Labele). These recordings were filed in a program for acquisition of biological signs, which was designed by the information and technology sector of InCor specifically for performing the tilt-table test.

The patients rested for $20 \mathrm{~min}$ in the horizontal dorsal decubitus position, and the gurney was then tilted in approximately $5 \mathrm{~s}$ until the $60^{\circ}$ angle was reached. The patients were observed for $40 \mathrm{~min}$ or less, until reaching the criteria of positivity of the test (a decrease in systolic blood pressure $>30 \mathrm{mmHg}$ or a drop in heart rate, or both, associated with presyncopal or syncopal symptoms, or both) ${ }^{7}$. According to the behavior of blood pressure and heart rate, the positive hemodynamic responses were classified as follows: 1) vasodepressive: a drop in blood pressure with no significant change in heart rate; 2) mixed: arterial hypotension accompanied by a drop in heart rate; 3 ) cardioinhibitory: cardiac asystolia (pause >3s) accompanied by arterial hypotension; 4) tachycardic postural syndrome: an increase in heart rate $>30 \%$ as compared with the baseline heart rate during the prolonged period of monitoring in the orthostatic decubitus position, or heart rate $>120 \mathrm{bpm}$ in the first $10 \mathrm{~min}$, accompanied by symptoms of postural intolerance, without triggering the neurocardiogenic reflex.

Positivity was calculated using the following ratio: number of patients with a positive test/total number of patients with a history of syncope. Inference was performed using the analysis of Fisher exact test, the chi-square test, and the Mann-Whitney test. We adopted the significance level of $5 \%(\alpha=0.05)$.

\section{Results}

Comparative data related to sex, number of previous syncopal episodes, and duration of the history are shown in table I. No significant difference was observed when comparing the 2 groups.

In regard to the positivity of the test, group I had a rate of $41.6 \%$ (with 15 positive tests), and group II had a rate of $50 \%$ (with 29 positive tests). The type of positive response predominating in both groups was the mixed response. Distribution of the hemodynamic responses is shown in table II, and no statistical difference was observed in this distribution.

The mean period of exposure required for the test to become positive was shorter in the group of children (11.0 $\pm 7.23 \mathrm{~min}-$ minimum of $2 \mathrm{~min}$ and maximum of $24 \mathrm{~min}$ ) as compared with that of the group of adolescents $(18.44 \pm 7.83 \mathrm{~min}-$ minimum of $2 \mathrm{~min}$ and maximum of $35 \mathrm{~min})$ $(\mathrm{p}=0.009)$.

No sedation was required for performing the test in any child. No complications occurred, and in all positive cases, the reflex was spontaneously reversed with repositioning of the gurney to the horizontal dorsal decubitus position, no intervention being necessary. Only rarely was the mother required to remain in the testing room to keep the child calm so the test could be performed.

\section{Discussion}

Based on this study, we observed that the positivity rate of the tilt-table test in children and adolescents with syncope of unknown origin was similar in both groups

\begin{tabular}{|c|c|c|c|}
\hline \multicolumn{4}{|c|}{$\begin{array}{l}\text { Table I - Comparative data between the groups of children and } \\
\text { adolescents in regard to age, sex, number of syncopal episodes, } \\
\text { and duration of the history }\end{array}$} \\
\hline Variable & Group & & \\
\hline Sex (female) & $\begin{array}{l}\text { Children } \\
20(55.5 \%)\end{array}$ & $\begin{array}{c}\text { Adolescents } \\
39(67.2 \%)\end{array}$ & $\mathrm{P}=0.279 *$ \\
\hline Age $\begin{array}{l}\text { minimum } \\
\text { maximum } \\
\text { (mean) }\end{array}$ & $\begin{array}{c}3 \text { years } \\
12 \text { years } \\
(9.19 \pm 2.31)\end{array}$ & $\begin{array}{c}13 \text { years } \\
18 \text { years } \\
(16.05 \pm 1.40)\end{array}$ & \\
\hline $\begin{array}{l}\text { Syncopal } \\
\text { episodes (mean) }\end{array}$ & $7.84 \pm 16.5$ & $6.18 \pm 8.17$ & $\mathrm{P}=0.859^{* *}$ \\
\hline $\begin{array}{l}\text { Duration } \\
\text { of history (months) }\end{array}$ & $29.43 \pm 29.65$ & $29.58 \pm 41.61$ & $\mathrm{P}=0.253^{* *}$ \\
\hline
\end{tabular}

\begin{tabular}{|c|c|c|}
\hline \multicolumn{3}{|c|}{$\begin{array}{l}\text { Table II - Distribution of the hemodynamic responses to the test in } \\
\text { the } 2 \text { groups }\end{array}$} \\
\hline \multirow[t]{2}{*}{ Type of response } & \multicolumn{2}{|c|}{ Groups } \\
\hline & Children & Adolescents \\
\hline Negative & $21(58.3 \%)$ & $29(50 \%)$ \\
\hline Mixed positive & $10(27.7 \%)$ & $18(31 \%)$ \\
\hline Positive vasodepressive & $4(11.1 \%)$ & $7(12 \%)$ \\
\hline Positive cardioinhibitory & $1(2.7 \%)$ & $1(1.7 \%)$ \\
\hline Tachycardic postural syndrome & $0(0 \%)$ & $3(5.1 \%)$ \\
\hline
\end{tabular}


(41.6\% and 50\%, respectively). In a previous study carried out at our institution and comprising 125 patients with a mean age of 34.6 years, the positivity rate was $41.6 \%$, which is very comparable to the results of the present study. Pavri et $\mathrm{al}^{8}$ analyzing data of 333 patients undergoing passive tilttable testing in 12 studies reported that the positivity rate of the test ranged from $7 \%$ to $55 \%$ with a mean of $24 \%$. Linzer et $\mathrm{al}^{9}$ in a study with 425 patients reported a positivity rate of the tilt-table test ranging from $26 \%$ to $90 \%$.

Since the initial studies by Kenny et al ${ }^{10}$ in 1986, tilt-table testing has shown effectiveness and safety in identifying patients with neurocardiogenic syncope, and, soon after, several investigators initiated its use in pediatric patients. However, the nonexistence of a gold standard and the lack of standardized protocols in regard to tilting angulation during the test, concomitant invasive procedures, administration of sensitizing pharmacological agents, and chronobiological factors, have made the determination of positivity and specificity difficult, resulting in great variations in the results obtained.

Pongiglione et al ${ }^{11}$, in a study comprising 20 patients (mean age of 12.5 years) with recurrent syncopes who underwent the passive tilt-table test at $60^{\circ}$ for $15 \mathrm{~min}$, followed by the sensitizing test with isoproterenol, reported an $80 \%$ positivity rate ( 16 patients). Of these, 4 patients had a positive test in the passive period and 12 patients after the use of isoproterenol. Thilenius et al ${ }^{12}$ reported a $74.2 \%$ positivity rate and reproduction in symptoms in $80 \%$ of the patients using the passive protocol at $60^{\circ}$ for $10 \mathrm{~min}$, followed by isoproterenol infusion in a total of 35 patients with ages ranging from 8 to 19 years. Alehan et al ${ }^{13}$, in a study comprising 20 patients (mean age of 12 years) undergoing the passive protocol at $60^{\circ}$ for $25 \mathrm{~min}$ followed by the sensitizing test with isoproterenol, obtained a $75 \%$ positivity rate, and only $25 \%$ of the patients had a positive test in the passive period. Later, in another study ${ }^{14}$, the same investigators performed the tilt-table test with the sensitized protocol or the passive type at $60^{\circ}$ for $45 \mathrm{~min}$. They studied 65 patients and $30 \mathrm{con}$ trols with mean ages of 11.4 and 12.8 years in the 2 groups, and they aimed to compare the sensitivity and specificity of the tilt-table test with and without isoproterenol. They reported positivity and specificity rates of $76.6 \% / 86.7 \%$ and $48.5 \% / 93.4 \%$, respectively, for the 2 groups. Grubb et al ${ }^{15}$ studied 30 patients who had had at least 3 episodes of syncope in the last 6 months, 14 years being their mean age, and used the passive protocol with a tilt angle of $80^{\circ}$ for $30 \mathrm{~min}$. The protocol was followed by the sensitizing test with isoproterenol. They reported a $70 \%$ positivity rate for the tilttable test and only $20 \%$ of positivity in the passive period. Using the same protocol in 66 patients with mean age of 13.6 years, Berkowitz et al ${ }^{16}$ reported a $65 \%$ ( 43 patients) positivity in the passive period with an addition of $12 \%$ in 20 of the 23 patients later undergoing the sensitizing test with isoproterenol. In this same study, the false-positive rate was $52 \%$ in the passive test. On the other hand, when sensitization with isoproterenol was used, no increase in the falsepositive rate was observed. The results led the authors to question the specificity of the test, and, at the same time, to recommend tests lasting up to $20 \mathrm{~min}$ to minimize the incidence of false-positive results.

In studies performed with no sensitizing agents, the results showed a lower positivity rate. Lerman-Sagle et al ${ }^{17}$, in a study with 15 patients (mean age of 14.4 years) and 10 controls, performed the tilt-table test at $60^{\circ}$ for $60 \mathrm{~min}$ and obtained a $43 \%$ positivity rate with $100 \%$ specificity. Fouad et al ${ }^{18}$ provoked a vasovagal response in 25 out of 45 patients (57\%), and in 3 out of 18 controls (17\%). In addition to the tilt-table test, some investigators have used the orthostatic test based on the same principle as the tilt-table test for assessing syncope. Ross et al ${ }^{19}$, using a protocol in which the patients, after a resting period, remained in the orthostatic position at $90^{\circ}$ without any support for $12 \mathrm{~min}$, obtained syncope in $44 \%$ of 104 patients (mean age $=13$ years). Oslizlok et al ${ }^{20}$, however, with the same tilting angulation but for $15 \mathrm{~min}$, reported a positivity rate of $58 \%$ in 209 patients, whose ages ranged from 1.8 to 18 years.

Specificity in regard to different tilting angles was the objective of the study by Lewis et al ${ }^{21}$. In this study, the controls, whose ages ranged from 12 to 18 years, underwent the passive tilt-table test with angles of $60^{\circ}, 70^{\circ}$, and $80^{\circ}$, and a duration of $30 \mathrm{~min}$, which resulted in specificities of $69 \%, 71 \%$, and $40 \%$, respectively. On the basis of the results obtained, the authors recommended the use of angles of $60^{\circ}$ or $70^{\circ}$ for no longer than $10 \mathrm{~min}$. In another study with controls, whose ages ranged from 6 to 16 years, Van Steenwijk et $\mathrm{al}^{22}$ used the orthostatic test and the tilt-table test with a tilting angle of $70^{\circ}$. Of the 68 controls, $29(40 \%)$ had a falsepositive result, which was attributed to the associated intravascular procedure. Despite data presented, it is worth noting that the tilt-table test identifies an excessive susceptibility to the vasovagal reflex, to which all human beings are vulnerable, depending on the stimulus. Therefore, specificity of the tilt-table test may have been actually underestimated. In our study, the patients did not undergo intravascular procedures aiming to minimize false-positive results.

Most of the hemodynamic responses of the positive tests obtained in our study were mixed $(66.6 \%$ in children and $62.0 \%$ in adolescents). In regard to this parameter, data in the literature vary. Mangru et $\mathrm{al}^{23}$ and Müller et $\mathrm{al}^{24}$, studying patients whose mean ages were 14 and 13 years, respectively, reported results similar to ours, in which the mixed response predominated with $50 \%$ and $71.4 \%$, respectively. On the other hand, Alehan et al ${ }^{13}$ reported a vasodepressive response in $67 \%$ of patients, mixed in $27 \%$, and cardioinhibitory in $6 \%$. In regard to duration of exposure to the orthostatic decubitus position for a positive test, the results reported ranged from $8.5 \mathrm{~min}$ to $48 \mathrm{~min}$. No study separately analyzing children and adolescents has been reported. In a previous study carried out in our service and cited above, with patients whose mean age was 34.2 years, the time elapsed between tilting and the appearance of symptoms ranged from 3 to $40 \mathrm{~min}$, with a mean of $18 \mathrm{~min}$ and $25 \mathrm{~s}$. In the present study, the means obtained (11.0min for children and $18.4 \mathrm{~min}$ for adolescents) were similar to most data in the 
literature, with earlier responses for children as compared with those for adolescents.

In conclusion, the tilt-table test is a useful method for assessing unexplained syncope in children, and no difference was observed in the positivity rates for children and adolescents. The predominant hemodynamic response of the positive tests was mixed in both groups. Children had earlier positive responses as compared with those of adolescents.

\section{References}

1. Prodinger RJ, Reisdorff EJ. Syncope in Children. Emerg Med Clin North Am 1998; 16: 617-26.

2. McHarg ML, Shinnar S, Rascoff H, Walsh CA. Syncope in childhood. Pediatr Cardiol 1997; 18: 367-71.

3. Driscoll DJ, Jacobsen SJ, Porter CJ, Wollan PC. Syncope in children and adolescents. J Am Coll Cardiol 1997; 29: 1039-45.

4. Lewis DA, Dhala A. Syncope in the pediatric patient. Pediatr Clin North Am 1999; 46: 205-19.

5. Benditt DG, Ferguson DW, Grubb BP, et al. Tilt table testing for assessing syncope. J Am Coll Cardiol 1996; 28: 263-75.

6. Tanel RE, Walsh EP. Syncope in the pediatrics patient. Cardiol Clin 1997; 15: 277-94.

7. HachulD, Sosa EA, Consolim F, et al. Valor diagnóstico do teste de inclinação na avaliação da síncope de origem indeterminada. Resultados preliminares. Arq Bras Cardiol 1994; 62: 7-9.

8. Pavri BB, Ruskin JN, Brooks R. The yield of head-up tilt testing is not significantly increased by repeating the baseline test. Clin Cardiol 1996; 19: 494-6.

9. Linzer M, Yang EH, Mark Estes III NA, Wang P, Vorperian VR, Kapoor WN Diagnosing syncope part 2: Unexplained syncope. Ann Intern Med 1997; 127: 76-86

10. Kenny RA, Ingram A, Bayliss J, Sutton R. Head-up tilt: A useful test for investigating unexplained syncope. Lancet 1986; 11: 1352-4.

11. Pongiglione G, Fish FA, Strasburger JF, Benson W. Heart rate and blood pressure response to upright tilt in young patients with unexplained syncope. J Am Coll Cardiol 1990; 16: 165-70.

12. Thilenius OG, Quinones JA, Husayni TS, Novak J. Tilt test for diagnosis of unexplained syncope in pediatric patients. Pediatrics 1991; 87: 334-8.

13. Alehan D, Celiker A, Özme S. Head-up tilt test: a highly sensitive, specific test for children with unexplained syncope. Pediatr Cardiol 1996; 17: 86-90.

14. Alehan D, Lenk M, Özme S, Celiker A, Özer S. Comparison of sensitivity and spe- cificity of tilt protocols with and without isoproterenol in children with unexplained syncope. PACE 1997; 20: 1769-76.

15. Grubb BP, Temesy-Armos P, Moore J, Wolfe D, Hahn H, Elliott L. The use of headupright tilt table testing in the evaluation and management of syncope in children and adolescents. PACE 1992; 15: 742-8.

16. Berkowitz JB, Auld D, Hulse E, Campbell RM. Tilt table evaluation for control pediatric patient: Comparison with symptomatic patients. Clin Cardiol 1995; 18: 521-5.

17. Lerman-Sagle T, Rechavia E, Strasberg B, Sagie A, Blieden L, Mimouni M. Headup tilt for the evaluation of syncope of unknown origin in children. J Pediatr 1991; 118: 676-9.

18. Fouad FM, Sitthisook S, Vanerio G, et al. Sensitivity and specificity of the tilt table test in young patients with unexplained syncope. PACE 1993; 16: 394-400.

19. Ross BA, Hughes S, Anderson E, Gillette PC. Abnormal responses to orthostatic testing in children and adolescents with recurrent unexplained syncope. Am Heart J 1991; 122: 748-54.

20. Oslizlok P, Allen M, Griffin M, Gillette P. Clinical features and management of young patients with cardioinhibitory responses during orthostatic testing. Am J Cardiol 1992; 69: 1363-5.

21. Lewis DA, Zlotocha J, Henke L, Dhara A. Specificity of head-up tilt testing in adolescents: eddect of various degrees of tilt challenge in normal control subjects. J Am Coll Cardiol 1997; 30: 1057-60.

22. Van Steenwijk CCEJV, Wieling W, Johannes JM, Harms MP, Kuis W, Wesseling $\mathrm{KH}$. Incidence and hemodynamic characteristics of near-fainting in healthy 6- to 16- year old subjects. J Am. Coll Cardiol 1995; 25: 1615-21.

23. Mangru NN, Young ML, Mas MS, Chandar JS, Pearse LA, Wolff GS. Usefulness of tilt table test with normal saline infusion in management of neurocardiac syncope in children. Am Heart J 1996; 131: 953-5.

24. Müller G, Deal BJ, Strasburger JF, Benson DW. Usefulness of metoprolol for unexplained syncope and positive response fo tilt testing in young persons. Am J Cardiol 1993; 71: 592-5. 